\title{
Belgeo
}

Revue belge de géographie

3 | 2014

Art(s) \& Espace(s) / Art(s) \& Space(s)

\section{La nature comme œuvre d'art: Élisée Reclus et les (néo)impressionnistes}

Nature as a work of art: Élisée Reclus and the (neo)impressionists

\section{Federico Ferretti}

\section{(2) OpenEdition}

\section{Journals}

Édition électronique

URL : http://journals.openedition.org/belgeo/13207

DOI : 10.4000/belgeo.13207

ISSN : 2294-9135

Éditeur :

National Committee of Geography of Belgium, Société Royale Belge de Géographie

Référence électronique

Federico Ferretti, «La nature comme œuvre d'art: Élisée Reclus et les (néo)impressionnistes », Belgeo [En ligne], 3 | 2014, mis en ligne le 19 décembre 2014, consulté le 30 avril 2019. URL : http:// journals.openedition.org/belgeo/13207; DOI : 10.4000/belgeo.13207

Ce document a été généré automatiquement le 30 avril 2019

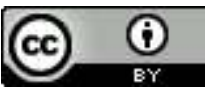

Belgeo est mis à disposition selon les termes de la licence Creative Commons Attribution 4.0 International. 


\title{
La nature comme cuvre d'art: Élisée Reclus et les (néo)impressionnistes
}

\author{
Nature as a work of art: Élisée Reclus and the (neo)impressionists
}

\author{
Federico Ferretti
}

\section{NOTE DE L'AUTEUR}

Cette recherche a été financée par le Fonds National Suisse pour la recherche scientifique (FNS) dans le cadre des projets Écrire le Monde Autrement : géographes, ethnographes et orientalistes en Suisse romande, 1868-1920, des discours hétérodoxes [FNS div. 1, 2012-2015] et Géographie, éducation publique et pédagogie libertaire en Suisse et en Europe (19e-21 ${ }^{e}$ siècle) [FNS div. Interdisciplinaire, 2014-2017].

\section{Introduction}

1 Dans sa longue carrière de géographe et de militant, Élisée Reclus (1830-1905) a été concerné par les arts figuratifs, et notamment par la peinture, sous trois aspects au moins. Le premier, sa collaboration avec plusieurs artistes pour la construction du riche appareil iconographique de ses ouvrages. C'est le cas de Charles Perron (1837-1909) et André Słomczynski (1844-1910), chargés respectivement des cartes et des dessins de la Nouvelle Géographie universelle; de Léon Benet (1839-1916), dessinateur de Jules Verne, qui a illustré Histoire d'une montagne et Histoire d'un ruisseau; de František Kupka (1871-1957), l'un des pères de l'abstraction, qui a illustré L'Homme et la Terre. Le deuxième, sa fréquentation directe avec des peintres qui partageaient ses démarches scientifiques et politiques, notamment Gustave Courbet (1819-1877), militant de la Commune de Paris de 1871, Camille Pissarro (1830-1903), sympathisant anarchiste, Auguste Baud-Bovy (1848-1899), participant des réseaux de sociabilité de Reclus et de ses collègues en Suisse. Le troisième, son influence sur des peintres néo-impressionnistes qui se sont inspirés à la 
fois de sa géographie et de son anarchisme, comme Paul Signac (1863-1935) et Maximilien Luce (1858-1941).

Reclus côtoie des genres artistiques différents, en se considérant intéressé plutôt aux contenus et usages sociaux de l'art qu'aux langages visuels en-soi, et pourtant participe à la mise en place d'une esthétique de ses propres savoirs spatiaux. Son époque est considérée par les historiens et par les critiques comme la période où commencent les débats sur l'art social, qui incite les artistes à sortir de leurs cabinets pour faire circuler leurs idées dans la société, en vue d'un changement social (Prochasson, 2006).

Cet article se situe dans le cadre de la littérature récente, francophone et internationale, qui aborde les relations historiques et épistémologiques entre géographie et anarchisme en tant que pensées également concernées par la transformation du monde à partir de ses spatialités (Pelletier, 2013; Springer, 2013). Nous retenons plus spécifiquement la littérature qui a analysé les travaux d'Élisée Reclus du point de vue de ses réseaux politiques, scientifiques et familiaux (Brun, 2014 ; Ferretti, 2014a), en nous inspirant de la théorie de l'acteur-réseau de Bruno Latour (1987) pour l'appliquer à un épisode non institutionnel de l'histoire de la géographie, qui a pourtant contribué de manière significative à la formation de la géographie contemporaine.

Notre analyse se construit autour des réseaux reclusiens, en les interrogeant du point de vue de la participation d'artistes et de la signification de l'art pour les propositions scientifiques et militantes des acteurs impliqués. Comment Reclus et ses collaborateurs utilisent-ils l'art dans leurs stratégies de communication scientifique et leur appréhension de l'espace? Quels sont les concepts et les savoirs géographiques mis en place par les artistes concernés, s'inspirant à la fois des paysages, des descriptions de terres éloignées et des thèmes sociaux mobilisés par la géographie? Quels sont les usages sociaux de ce transfert interdisciplinaire entre l'art et la géographie?

5 En partant de notre propre acquis de recherche et en nous appuyant sur les travaux des historiennes de l'art Robyn Roslak et Marina Ferretti-Bocquillon, nous allons interroger la relation entre Reclus et les mouvements néo-impressionnistes et modernes sur les questionnements exposés, en structurant l'article en trois parties.

Dans la première, nous abordons les origines proudhoniennes de l'idée de l'art porteur d'une valeur politique et pédagogique, pour comprendre quel rôle cette idée a joué dans la formation des idées de Reclus et de ses collègues, notamment pendant leur exil suisse; dans la deuxième, nous analysons le concept reclusien du monde comme œuvre d'art, en tant que dispositif relevant à la fois d'une préoccupation heuristique et d'une rhétorique militante, s'appuyant sur la double proposition d'éduquer au beau à travers la nature et de construire pour cela des objets tridimensionnels comme globes et reliefs; dans la troisième, nous interrogeons les réseaux de sociabilité qui unissaient anarchistes et (néo)impressionnistes et les façons dont ces derniers ont pu se nourrir des savoirs géographiques et soutenir parfois la diffusion des idées anarchistes.

\section{L'art pour le peuple : inspiration proudhonienne et réseaux d'exil}

7 L'approche de Reclus de l'art et les relations que le géographe anarchiste entretient avec peintres et dessinateurs s'inspirent du débat sur « l'art et le peuple » qui avait été lancé 
par l'un des inspirateurs à la fois de son anarchisme et de sa géographie (notamment à travers l'idée de fédéralisme), Pierre-Joseph Proudhon (1809-1865).

Ce dernier intervient sur les polémiques qui avaient investi, dans les années 1860, la peinture « réaliste » de Gustave Courbet, proposant un « discours sur l'art démocratique » (Schlesser, 2005). Cela consiste d'abord dans un refus de la métaphysique et de l'art religieux, pour pratiquer ce qu'on définira plus récemment comme un "art social» (Prochasson, 2006), ayant comme finalité l'émancipation du peuple. L'art de Courbet, à partir de tableaux comme Un enterrement à Ornans, vise un but philosophique et social; d'après Thomas Schlesser, le peintre "attribue d'abord à son art une fonction philosophique ('l'émancipation de la raison') et, ce faisant, influe sur le cours social et politique en promouvant la libération individuelle puis, à travers la démocratie, la libération collective » (Schlesser, 2005). La substitution de la réalité à « l'illusion mystique et religieuse » devient donc un enjeu pédagogique pour un artiste qui se considère issu du peuple et qui « s'adresse directement au peuple : 'l'heure n'est plus à peindre les nantis, l'avenir est à l'art social' » (ibid.). Toujours selon Schlesser, le bouleversement par Courbet des hiérarchies établies parmi les personnages traditionnellement représentés dans les tableaux, participe de la même démarche.

Déjà en 1854, Courbet écrivait à un ami à propos de Proudhon: «je voudrais bien avoir aussi ce philosophe Proudhon qui est de notre manière de voir, s'il voulait poser j'en serais content » (Courbet, 1996, p. 122).

Figure 1. G. Courbet, Pierre-Joseph Proudhon et ses enfants, 1853, Paris, Musée du Petit Palais.

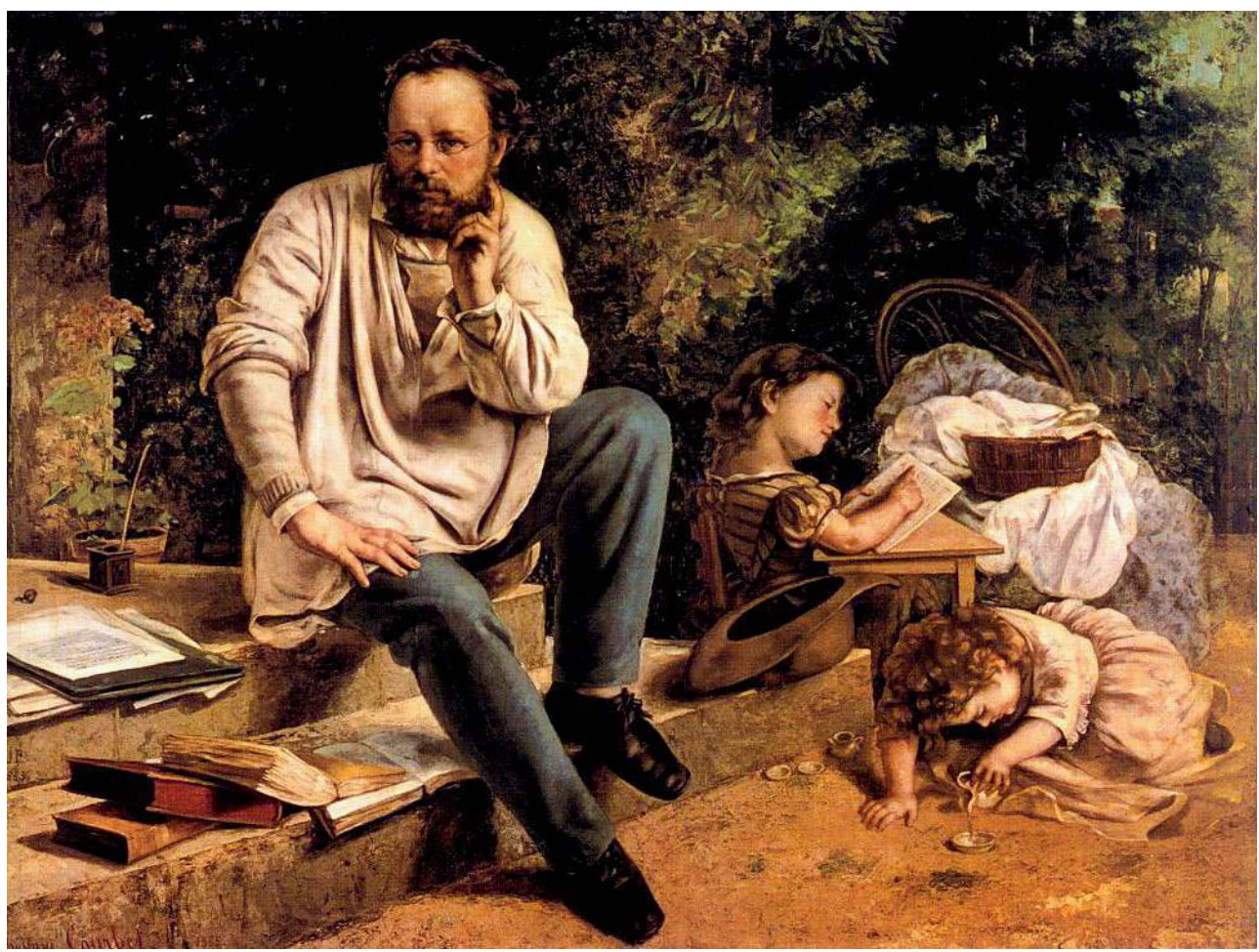

Copyright de l'image : WikiArt.org

10 Proudhon, de son côté, consacre un important travail, Du principe de l'art et de sa destination sociale, au rôle que l'esthétique doit jouer, selon lui, dans le processus d'émancipation sociale. Il considère l'art comme un enjeu politique à partir de la succession des écoles, 
parmi lesquelles il envisage une tendance classique représentée par Ingres, une tendance romantique représentée par Delacroix et une école réaliste représentée par Courbet. C'est l'admission d'Ingres au Sénat décidée par les notables du Second Empire qui fait dire à Proudhon : «Comment donc, encore une fois, le gouvernement préfère-t-il, en fait d'art, la décrépitude à la jeunesse, les antiquailles aux inventions nouvelles? L'art est-il un élément de civilisation ou de décadence?» (Proudhon, 1875, p. 8). Selon le philosophe franc-comtois, la réponse est qu'il n'existe pas un art neutre: "Toute création de l'art, comme de l'industrie ou de la politique, a nécessairement une destination: elle est faite pour un but. Il est absurde de supposer que quelque chose se produise dans la société à seule fin de se produire» (ibid., p. 369). Il y a donc deux alternatives pour l'art: la première est qu'il se fasse véhicule de l'émancipation sociale à travers son réalisme et sa valeur éducative, "en vue du perfectionnement physique, intellectuel et moral de l'humanité » (ibid., p. 370) ; la deuxième est que, «pour n'avoir pas voulu d'une mission hautement morale, pratique et positive, l'école de l'art pour l'art s'en donnera une parfaitement irrationnelle, chimérique et immorale » (ibid., p. 370).

11 C'est dans ce cadre politique et culturel qu'il faut lire les étroites relations professionnelles et amicales que le géographe Reclus entretient avec des artistes, surtout peintres et dessinateurs. Courbet partage avec le géographe anarchiste la participation à la Commune de 1871, où il est délégué à la culture, et l'exil successif en Suisse romande, où les deux Français se côtoient, sans qu'il y ait entre eux une collaboration stable, puisque Reclus n'arrive dans le Vaud qu'en 1874 et Courbet y meurt en 1877.

Cependant, les deux hommes ont au moins un collaborateur commun, le peintre polonais André Słomczynski, combattant de la Commune, proche de Courbet chez lequel il habite pendant cette période (Lindsay, 1973, p. 308), et ensuite collaborateur de la Nouvelle Géographie universelle de Reclus, pour laquelle il réalise une partie des dessins pour les gravures hors-texte (Reclus, 1927).

Exilé en Suisse de 1872 à 1890, Reclus noue des relations durables avec des artistes helvétiques, à partir de sa rencontre avec Auguste Baud-Bovy, figure originale, membre de ce que l'historien Marc Vuilleumier a défini comme "une colonie» d'artistes et artisans genevois qui dès 1849 "avait acquis le château de Gruyères où ses membres passeront désormais une partie de la belle saison, restaurant les bâtiments fort délabrés [...]. Courbet passera lui aussi par Gruyères, en 1855 et plus tard. Cette ouverture sur le monde extérieur [l'accueil à des militants et artistes étrangers N.d.A.] apparaît également dans les conceptions politiques et sociales de la plupart des membres " (Vuilleumier, 1996, p. 13). Au radicalisme de la première génération des animateurs de ce " phalanstère genevois » (Anker, 1991, p. 22) suivra le socialisme de la suivante : Baud-Bovy s'intéresse aux réunions de l'Internationale qui ont lieu à Genève dans les années 1860, et depuis 1871 on le considère «le protecteur dévoué en Suisse de tous les réfugiés de la Commune » (ibid., p. 22), parmi lesquels Courbet, Reclus, le célèbre communard Benoît Malon et sa compagne André Léo, pseudonyme de Léodile Béra-Champseix, romancière et féministe, amie des frères Reclus et protagoniste avec Baud-Bovy d'une longue correspondance (Vuilleumier, 1996).

14 C'est le peintre genevois qui implique Élisée Reclus dans les conférences populaires qui ont lieu à Genève à partir de 1875 (Heimberg, 1996, p. 544). La correspondance entre les deux hommes est révélatrice de l'importance de l'éducation dans leurs démarches à la fois politiques et scientifiques, ce qui se répercute dans leurs conceptions respectives de l'art. Baud-Bovy est d'abord l'un de ceux qui aident Reclus, résidant de 1872 à 1874 dans 
un village isolé du Tessin, à se procurer les livres nécessaires à l'entreprise de la Nouvelle Géographie universelle, en lui servant de relais auprès de la Société de Géographie de Genève. « Madame André Léo a eu la bonté de vous écrire à mon sujet, ce qui m'enhardit à vous écrire moi-même. Les livres m'arrivent assez difficilement, et bien souvent j'envoie lettre sur lettre à des libraires pour pouvoir obtenir les ouvrages que je demande. Ainsi, je n'ai pu réussir à me faire envoyer le Globe, journal géographique de Genève [...]. Ne pourriez-vous, lorsque vous avez l'occasion d'aller de ce côté, m'abonner directement en vous adressant au secrétaire même de la Société de Géographie? »1

La lettre d'acceptation de Reclus pour les conférences populaires révèle l'importance attribuée à l'éducation et le constant mélange de science et politique qui caractérise sa démarche.

Quoique occupé très au-delà de mes forces, je n'ose absolument pas refuser la proposition que vous me faites. L'idée de faire un cours à des ouvriers me séduit beaucoup, et je serais fort heureux de pouvoir être ainsi utile à notre cause. Mais le pourrais-je ? [...] Je vous prie donc de vouloir bien me donner plus de détails avant de proposer mon nom aux suffrages de la commission. $1^{\circ}$ Pourrai-je me borner à faire un cours par quinzaine ? $2^{\circ}$ Le cours choisi par moi, La Géographie appliquée à $l^{\prime}$ Histoire, conviendrait-il à ces messieurs [...] ? $3^{\circ}$ Aurai-je entière liberté de parole ? $4^{\circ}$ Pourrai-je discuter avec ces messieurs le choix du jour afin que je puisse profiter de mon voyage à Genève pour assister la veille ou le lendemain aux séances de la Société de Géographie ?

C'est toujours à Genève, dans les milieux des Internationalistes, que Reclus rencontre celui qui sera l'un de ses plus importants collaborateurs, auquel il confiera le dessin des cartes pour la Nouvelle Géographie universelle et ensuite la réalisation d'une partie de ses objets tridimensionnels (Ferretti, 2014b). Il s'agit de Charles Perron, peintre sur émail qui devient cartographe à l'époque où les « systèmes iconographiques » de cartes, tableaux, photos, dessins et gravures s'affirment dans l'édition géographique (Mendibil, 2000).

Dans les archives du géographe, le lien étroit qu'il envisage entre art et éducation est confirmé par ses correspondances avec le fils d'Auguste, Daniel Baud-Bovy (1870-1958), écrivain et critique d'art. Dans ses lettres à Reclus, ce dernier lamente la mort de Barthélemy Menn (1815-1893), artiste et sorte de guru spirituel du groupe genevois Les Humanistes. Les membres de ce cénacle de disciples de Menn, parmi lesquels le jeune Baud-Bovy, visaient à « une transformation des principes régulateurs de l'éducation par le moyen de la peinture » (Anker, 1991, p. 68). D. Baud-Bovy écrit à Reclus que sans Menn, il serait désormais impossible de réaliser son projet d'une école d'art « autogérée » dans une ville conformiste comme Genève. ${ }^{3}$

ans les années de l'exil suisse, Reclus correspond avec Pierre-Jules Hetzel au sujet de l'engagement du dessinateur de Jules Verne, Léon Benet, dans l'illustration de l'Histoire d'un ruisseau et l'Histoire d'une montagne. Le géographe échange avec l'éditeur plusieurs lettres où il expose son idée d'une iconographie qui puisse soutenir la tâche didactique du texte, tout en restant fidèle à la réalité du paysage et à la "rigueur scientifique » (Ferretti, 2012b).

19 Reclus et les artistes directement impliqués dans son œuvre, à ce moment, partagent généralement une idée de l'art social fortement didactique et assez réaliste-descriptive, ce qui ne sera pas toujours le cas, comme on le verra, chez leurs amis les néoimpressionnistes. dernier travail, L'Homme et la Terre, car cet ouvrage paraît en grande partie à titre 
posthume. Ce n'est que le 4 juin 1905, un mois avant la mort du géographe, que Kupka, l'auteur des riches illustrations « intimement liées aux textes » (Salé, 2002, p. 105) des six volumes, lui envoie les premières deux images : il s'agit de la couverture et du célèbre carré contenant l'exergue "l'homme est la nature prenant conscience d'elle-même. »" Cependant, d'après les recherches de Marie-Pierre Salé dans les archives de Kupka, les deux hommes avaient eu l'occasion de se rencontrer et d'échanger à ce sujet dans les mois précédents, en entamant une « affectueuse collaboration » (ibid, p. 105).

Figure 2. L'exergue de L'Homme et la Terre, rappelant les principes de la Naturphilosophie.

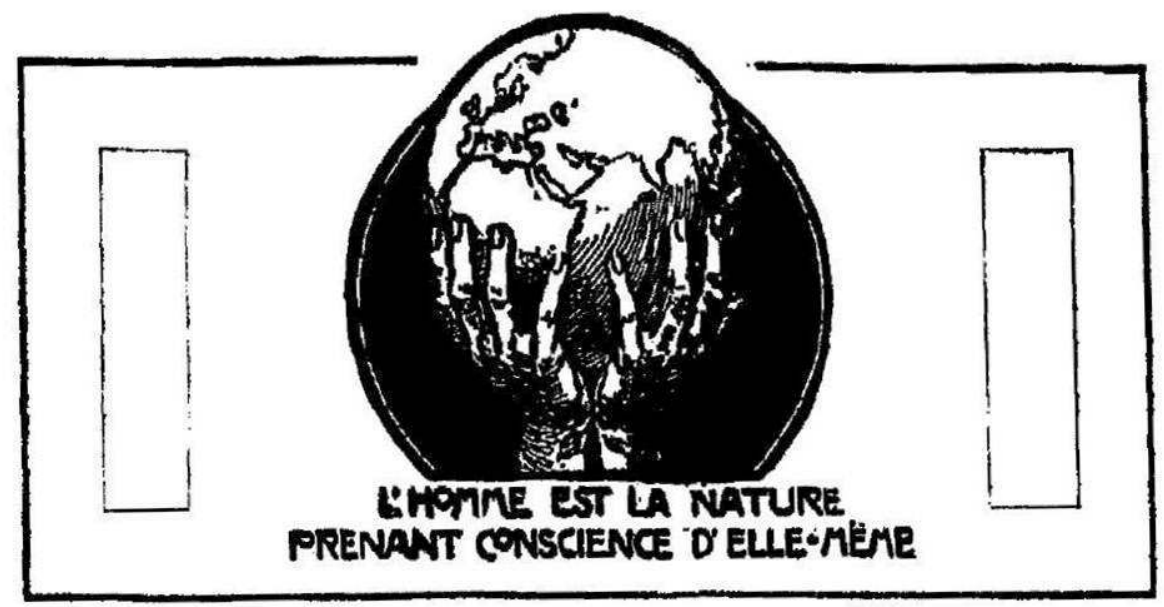

\section{Globes, plein air et philosophie de la nature : le monde comme œuvre d'art}

L'exergue illustré à la figure 2 nous renvoie à un point primordial des conceptions esthétiques et éthiques de Reclus : sa référence constante à la philosophie de la nature, plus précisément à la Naturphilosophie allemande de Friedrich Schelling (1775-1854) et Lorenz Oken (1779-1851), auteurs qui ont participé de sa formation juvénile (Reclus, 1911, p. 17), tout comme Baruch Spinoza (1632-1677), influent sur Schelling et Oken ainsi que sur Reclus lui-même (Ferretti, Malburet et Pelletier, 2011).

Cette approche, considérant humanité et environnement comme des entités consubstantielles, refuse toute scission à priori entre nature et culture, entre naturel et artificiel, entre histoire et milieu. Ce qui en découle pour Reclus est que la vraie œuvre d'art doit être le monde, mais non celui de l'état de nature de Rousseau, ni la nature vierge de géographes contemporains comme l'Américain George Perkins-Marsh (1801-1882), attiré plutôt par la wilderness.

23 Le monde doit être embelli par l'humanité à travers son travail, lequel ne peut s'accomplir, d'après Reclus, que par une transformation de la société. Comme le géographe déclare dans son écrit le plus explicite sur cette question, L'Art et le Peuple :

A la fermeture du Salon, un de mes amis, grand amateur de belles choses, m'arriva tout désolé. Il avait été malade, puis un voyage l'avait éloigné de Paris ; maintenant il revenait trop tard pour visiter l'Exposition [...] Qu'il se rassure, le cher compagnon! Une promenade dans les sentiers de la forêt, sur les feuilles froissées, 
ou bien une minute de repos au bord d'une fontaine pure, - s'il s'en trouve encore à quinze ou vingt lieues du boulevard - le consoleront d'avoir manqué sa visite au palais coutumier où, tous les ans, sont enfermés temporairement ce que l'on appelle les « beaux-arts » (Reclus, 1904, éd. 2012, p. 140). émerveillement les œuvres exposées dans ces salons, tout en se posant des questions à propos de leur signification et de leur utilité. «Là aussi je vois des artistes prestidigitateurs qui manipulent et mélangent les couleurs avec une incomparable dextérité, qui marient de mille façons les ombres et les clairs en des ragoûts tout à fait inattendus et réussissent à faire surgir des fonds noirs une lumière étourdissante. Tout cela me paraît vraiment fort beau, ou plutôt surprenant, et j'applaudis les virtuoses du pinceau en toute sincérité. Et cependant je ne suis point satisfait. Est-ce bien cela qui est l'art véritable? » (ibid., p. 141).

La première critique est de caractère social : « Est-ce que tous ces objets peints, sculptés, gravés ou brodés peuvent me faire oublier la sordide misère du dehors, et la présence du sergent de ville armé qui, là-bas, près de la porte, ou dans la salle même, pourra braquer une arme sur le citoyen paisible et lui briser le crâne ? Non, tout cet art polychrome qui accumule ses produits disparates dans les salles dorées que prête l'État, ne peut être qu'un art faux, mensonger, car il n'est pas l'œuvre d'un peuple libre » (ibid., p. 141).

Un problème qui en ressort est celui de la liberté de l'artiste dans la société capitaliste, et aussi celui de la nécessité de célébrer, dans le cas de la peinture réaliste-historique qui s'était affirmée avant l'impressionnisme, les gestes des rois et l'histoire évènementielle : « les images des faux grands hommes y pullulent » (ibid., p. 141).

Par contre, un art libre pourrait commencer à embellir la planète, d'après le géographe, à partir de l'architecture et de ce qu'aujourd'hui on appelle l'aménagement. «Ah! Si les peintres et les sculpteurs étaient libres, ils n'auraient pas besoin de s'enfermer en des salons. Ils n'auraient qu'à reconstruire nos cités ; tout d'abord à démolir ces ignobles cubes de pierre où se sont entassés les êtres humains » (ibid. p. 141-142).

Le principe reclusien de la beauté se mêle sans cesse à celui de justice. En réinterprétant des clichés de son siècle, le géographe retrace dans l'histoire des exemples de participation collective à la construction de bâtiments qui respectaient à la fois la liberté des travailleurs et la beauté du résultat. « De cette manière on vit surgir les temples des villes helléniques et les nefs du moyen âge : des populations entières, animées d'un même esprit, entraînées d'un même désir, collaboraient à l'œuvre commune qui devait être à la fois la gloire de tous et la joie particulière de chacun des citoyens ; celui-ci reconnaissant la pierre apportée par lui, tel autre, le bas-relief qu'il avait sculpté ou la couleur due à son pinceau. Pas un communier qui ne retrouvât, dans le bel ensemble de l'édifice, la partie où son propre idéal de beauté avait pris sa forme matérielle » (ibid. p. 143).

Mais pour réaliser tout cela, on ne peut passer sous silence les conditions matérielles de la production artistique, ni l'incompatibilité entre art et misère: "Comment un peuple deviendrait-il artiste quand les souffrances de la faim et de la maladie forcée l'enlaidissent ?» L'art, dit Reclus en citant Ruskin, doit embellir le peuple; le géographe ne fait pas question de style, ce qu'il aime de l'art est son engagement social. «L'Art c'est la vie, dit Jean Baffier, l'ouvrier sculpteur qui a mis tant de passion et de joie à tailler dans le marbre la noble et pure figure de la paysanne, sa mère, et celle des vaillants laboureurs, des prudents jardiniers ». 
30 Finalement, l'approche reclusienne de l'art libre comme embellissement du monde ne se limite pas à l'architecture urbaine, en vertu aussi de son idée de l'intégration progressive entre ville et campagne (Ferretti, 2012), voire entre humanité et nature (de manière cohérente avec les présupposés de la Naturphilosophie). "Ce n'est pas seulement la restauration, l'embellissement de nos villes que nous attendons de l'homme devenu artiste, parce qu'il sera devenu libre, nous comptons aussi sur lui pour qu'il renouvelle la beauté des campagnes en adaptant toutes ses œuvres propres au milieu de la nature » (Reclus, 1904, éd. 2012, p. 146). Les exemples sont tirés encore de l'histoire, d'une époque où, selon le géographe, la cohérence entre un édifice et le site environnant n'échappait pas à ses constructeurs: "C'est ainsi qu'un temple grec continue, développe et fleurit, pour ainsi dire, les contours du rocher sur lequel on l'a dressé ; il en fait partie intégrante, mais il lui donne un sens plus élevé » (ibid., p. 147).

31 Les exemples contraires relèvent des problèmes liés à la spéculation, qui semblent garder aujourd'hui une certaine actualité: "L'on ressent une impression de véritable dégoût lorsque d'insolents architectes, payés par des hôteliers sans pudeur, bâtissent d'énormes caravansérails, blocs rectangulaires où sont inscrits mille rectangles de fenêtres symétriques et que hérissent cent cheminées fumantes, le tout en face de pics superbes de granit, des champs de neige immaculés, des fleuves de glace bleue serpentant dans les vallées de la montagne » (ibid., p. 147).

La conclusion unissant pour le même but art, géographie et politique ne pourrait être plus claire : «La Terre est infiniment belle, mais pour nous associer à sa beauté, pour la glorifier par un art respectueux, il n'est d'autre moyen que de se rendre libre, de faire la révolution décisive contre l'argent et d'abolir la lutte des classes en abolissant les classes elles-mêmes » (ibid., p. 147-148).

La laideur, au contraire, surgit alors que l'équilibre entre humanité et nature est rompu par des établissements artificiels faits pour le profit, comme les grandes usines, ou pour la raison d'État, comme les villes militaires. On est clairement face à une stratégie communicative qui vise à la fois à la propagande politico-sociale et à la construction d'une science qui fait de l'esthétique l'un des éléments de son éthique, pour améliorer le monde à partir de sa géographie.

Dans ses premiers écrits géographiques, dont l'article «Du sentiment de la nature dans les sociétés modernes ", Reclus articule la notion problématique de l'environnement autour d'arguments de caractère esthétique. La beauté de la nature contraste avec la laideur de situations où "la spéculation s'empare de tous les sites charmants du voisinage, elle les divise en lots rectangulaires, les enclot de murailles uniformes, puis y construit par centaines et par milliers des maisonnettes prétentieuses [...] Sur le bord de la mer, les falaises les plus pittoresques, les plages les plus charmantes sont aussi en maints endroits accaparées soit par des propriétaires jaloux, soit par des spéculateurs qui apprécient les beautés de la nature à la manière des changeurs évaluant un lingot d'or " (Reclus, 1866, p. 377). La cause de cet enlaidissement est le fait que tout, là, « peut devenir propriété particulière » (ibid., p. 377) : on voit clairement le lien reclusien entre éthique et esthétique, apparentant la nature, la liberté et la justice au beau et la propriété privée à son contraire.

Chez Reclus, un autre volet des usages sociaux de l'art concerne l'éducation, notamment son idée de l'éducation géographique partant d'une expérience directe de la nature d'après la leçon de Pestalozzi (Ferretti, 2013). Dans L'Homme et la Terre, le géographe 
anarchiste aborde significativement l'art dans le chapitre consacré à l'éducation. « La part de l'éducation qui doit aboutir aux grandes transformations esthétiques est encore bien plus délicate que l'éducation scientifique, car elle est moins directe, et l'élaboration, toute personnelle, en est infiniment plus nuancée. L'impression de la beauté précède le sens du classement et de l'ordre : l'art vient avant la science. L'enfant est ravi de tenir dans sa main un objet lumineux, de couleur éclatante, aux tintements argentins ; il jouit délicieusement de la musique des nuances et des sons, et seulement plus tard il cherche à connaître le comment et le pourquoi de son hochet » (Reclus, 1908, pp. 479-480).

Cela ne signifie pas que l'art soit cantonné dans la catégorie de l'irrationnel et du nonscientifique. Au contraire, plus l'artiste est librement créatif, et plus le « vrai art » devient utile à la cause sociale. «Celui qui prétend le devenir par l'étude servile des maitres, par la mensuration et la reproduction précise des lignes tracées avant lui, par l'observation rigoureuse des règles posées antérieurement ne sera jamais qu'un lamentable copiste, un générateur de décadence et de mort. La première règle de l'art, ainsi que de toute vertu, est d'être sincère, spontané, personnel (Ruskin)» (ibid. p. 480). Le célèbre critique John Ruskin (1819-1900) est également mobilisé par un autre auteur libertaire comme Patrick Geddes, qui convoque son autorité pour affirmer que la connaissance esthétique précède la connaissance rationnelle, d'où l'importance de la promenade et de l'approche directe du monde dans l'enseignement de la géographie à partir de l'éducation primaire (Geddes, 1902).

37 Il n'est pas question, pour Reclus, de former des artistes en tant qu'intellectuels organiques du mouvement révolutionnaire, et aucune comparaison ne serait possible avec le réalisme soviétique des décennies suivantes : en vertu de leur critique de la séparation du travail manuel et du travail intellectuel (Kropotkine, 1910), les géographes anarchistes questionnent aussi la séparation entre artiste professionnel et public.

Ensuite, l'anticlérical Reclus, comme déjà Proudhon, ne ménage pas ses critiques envers l'art chrétien, son effet de persuasion et sa censure du corps humain.

La tyrannie matérielle des maitres et des castes n'est pas la seule qui empêche complètement, ou du moins, retarde le développement de l'art; la lourde oppression d'une opinion publique inintelligente produit le même résultat. Le mal accompli par l'hypocrisie religieuse et morale qui sévit dans les pays anglo-saxons sous le nom de cant est vraiment incalculable [...] Également en pays anglais, la littérature, les peintures dites " convenables » furent, jusqu'à une époque récente, forcées d'ignorer complètement la vie sexuelle, en dehors des élans de l'âme et du côté purement spirituel de l'amour il semblait que l'homme fût vraiment un être dépourvu de corps, une simple flamme, une lumière, un farfadet. À cet égard, la société moderne, toujours soumise à cette honte, à cette malédiction de la chair qu'avait prononcée le christianisme, est encore singulièrement inférieure à la noble Hellade, qui respectait et divinisait les formes humaines (Reclus, 1908, p. 484).

Reclus, qui compte pourtant au nombre des premiers propagandistes du nudisme, critique les nus d'atelier en les considérant comme quelque chose d'artificiel, et donc morbide. "Encore il est nécessaire que cette appréciation des corps, vivant dans la plénitude de leur vie, se fasse en des conditions de liberté complète, non par une suite de surprises ou bien dans l'atelier où des êtres habitués à une pose de convention se vendent à tant la séance » (ibid., p. 485). Et l'auteur d'ajouter une critique du marché et de la mode. "Or la mode règne encore, de même que règnent toujours le Seigneur Capital et les antiques survivances de l'Église et de l'État. Il n'est donc point à espérer que la mode, qui 
représente les intérêts d'innombrables fournisseurs et qui répond à un ensemble infini de petites passions personnelles, abdique de gré ou de force devant un régime nouveau d'art et de bon sens » (ibid., p. 490).

41 Mais Reclus ne fait pas uniquement de la théorie : après s'être occupé directement de l'appareil iconographique de ses ouvrages, il se lance dans la construction d'objets tridimensionnels de représentation du monde où l'aspect artistique et esthétique n'est pas prioritaire, mais demeure en tout cas présent. Il s'agit là de corriger les défauts des cartes bidimensionnelles qui, «d'autant plus fausses qu'elles s'appliquent à une partie plus considérable de la surface planétaire, ne peuvent que tromper le lecteur sur les dimensions relatives des régions différentes [tandis que] sur la rondeur d'un globe artificiel aucune méprise n'est possible au sujet de la superficie relative des diverses individualités terrestres » (Reclus, 1895a, pp. 3-4).

Figure 3. Esquisse du projet d'Élisée Reclus pour construire un Grand Globe à l'exposition universelle de 1900 à Paris.

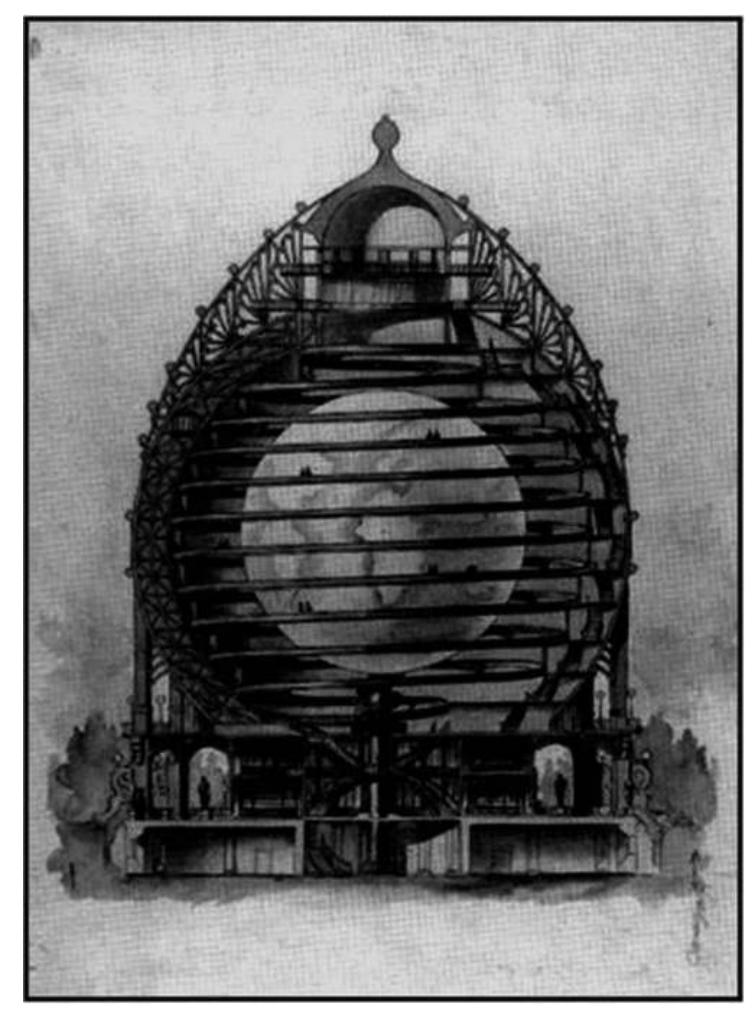

IFA, Fonds Louis Bonnier (dans Alavoine-Muller, 2003)

Reclus projette alors un grand globe au $100.000^{\mathrm{e}}$, c'est-à-dire de 127,5 mètres de diamètre, destiné à l'Exposition universelle de Paris de 1900. Nous ne reviendrons pas sur cette expérience, très étudiée (Alavoine-Muller, 2003; Dunbar, 1974; Ferretti, 2014b) sinon pour souligner trois points à notre avis fondamentaux : le premier, son insertion dans une tradition de globes, géoramas et jardins paysagers qui tout au long du $19^{\mathrm{e}}$ siècle a impliqué à la fois la géographie et les arts visuels (Besse, 2003) ; le deuxième, l'implication de néo-impressionnistes tels que Signac, Cross, Luce et Van Rysselberghe dans le décor pictural que Reclus envisageait pour la surface de l'énorme globe (Roslak, 2007, p. 109); le troisième, la grande puissance symbolique de cet objet comme tentative de rapprocher le 
plus possible le naturel et le construit, la réalité et sa représentation, bref de faire du monde une œuvre d'art.

Le rôle du géographe est alors celui d'accompagner le public, qu'il soit composé de jeunes ou d'adultes des classes populaires, dans sa prise de conscience du monde. L'aspect esthétique joue là un rôle heuristique important, qu'il soit à travers l'appréciation d'un objet-installation comme le grand globe, ou à travers l'approche directe à la vision des paysages par l'excursion et la promenade. Le savant crée, mais il doit surtout aider les autres à apprendre sans s'imposer. Cette maïeutique se double d'un volontarisme qui peut se passer de positions académiques et conditions matérielles : dans sa leçon inaugurale à l'Université Nouvelle de Bruxelles, établissement qui ne payait pas les professeurs et qui délivrait des titres non reconnus par l'État, Reclus affirme que "le savant doit être un héros » (Reclus, 1895b, p. 15). Finalement, on n'arrive pas à réaliser le Grand Globe, projet trop " engagé " pédagogiquement par rapport à la tradition plutôt spectaculaire des installations du même type (Alavoine- Muller, 2003 ; Besse, 2003).

Reclus entreprend alors, avec le cartographe belge Émile Patesson, la construction d'un Atlas Globulaire, constitué par des feuilles d'aluminium peintes et recourbées d'après « l'exacte courbure terrestre ", que le géographe présente à la Royal Geographical Society de Londres comme « a work of art, or of high-level artisanship » en concluant : «I speak here not as a geographer, but as an artist » (Reclus et al., 1903, p. 298). Dans les mêmes années, l'Institut de Géographie que Reclus avait fondé à l'Université Nouvelle reçoit la commande de ces matériaux pour l'École Moderne de Barcelone, fondé par Francisco Ferrer y Guardia, ${ }^{5}$ c'est-à-dire la plus célèbre parmi les expériences de la pédagogie libertaire de cette époque (Ferretti, 2013).

Tout au long du $20^{\mathrm{e}}$ siècle, plusieurs auteurs ont, eux aussi, proposé un lien entre art et éducation libertaire; parmi eux, il faut citer Herbert Read et son ouvrage Education through Art (1943). D'après Matthew Adams, Read rompt avec la tradition anarchiste, qui envisageait la révolution comme une rupture traumatique avec la société bourgeoise. « Read's view stood in distinction to the dominant strand in the historical tradition of anarchism, which generally looked forward to a cataclysmic sweeping away of the state " (Adams, 2013, p. 714).

Ce qu'Adams semble ne pas considérer (mais que Read ne pouvait pas ignorer) est qu'au contraire, dans l'histoire de l'anarchisme, la confiance dans une évolution graduelle de la structure sociale a été très forte exactement à l'époque de Reclus. Ce dernier considérait les concepts d'évolution et de révolution non comme antagonistes, mais comme passages également nécessaires de la transformation sociale (Reclus, 1898); le principal moteur de cette transformation aurait été exactement l'éducation dans laquelle, comme nous l'avons dit, l'art joue un rôle primordial. Malgré les lieux communs sur «barricades et dynamite » (Adams, 2013, p. 718) dans lesquels tombent aussi des commentateurs avertis comme Adams, des approches gradualistes ont aussi existé chez les anarchistes, notamment par des soutiens au volontarisme révolutionnaire développé par Errico Malatesta (Berti, 2003).

Même si Reclus n'est pas cité par Read, on peut supposer que ses idées arrivent à cet auteur à travers Kropotkine, très lu dans les pays anglo-saxons, et par William Morris (1834-1896), l'un des auteurs de référence de l'éducation artistique et socialiste à l'époque de Reclus, qui était en plus correspondant et ami de Kropotkine lui-même. ${ }^{6}$ Son 
mouvement réformateur Arts and Crafts semble d'ailleurs assez proche de l'art social tel qu'il était envisagé par les géographes anarchistes (Morris, 1884).

\section{Géographie libertaire et néo-impressionnistes} liens multiples qui existèrent entre les peintres néo-impressionnistes et l'anarchisme. Les chercheurs révèlent que, parmi les anarchistes impliqués, un rôle primordial a été joué par des géographes, en particulier Reclus et Kropotkine. Ce singulier rapprochement de l'art et de la géographie à travers l'anarchisme s'explique d'abord par les relations très étroites qui ont existé à cette époque entre l'idée anarchiste et la géographie sociale, et ensuite par le goût pour le paysage que des ouvrages géographiques très populaires comme ceux des géographes anarchistes contribuèrent à réveiller.

e le souligne Robyn Roslak, Paul Signac, dont l'adhésion à l'anarchisme est explicite, se déclare comme ayant été formé non seulement du point vue politique, mais aussi du point de vue culturel, par Reclus et Kropotkine (Roslak, 2006, p. 860). Plus encore, selon Roslak, anarchisme et néo-impressionnisme partagent des métaphores tirées de la chimie et des sciences naturelles, considérées alors comme sciences de la transformation sociale, de même que la géographie.

Chemical language and metaphors appeared regularly in the political commentaries of Grave and Kropotkin most often in their description of human societies [...]. The anarchist version of utopia, in common with Fénéon description of the idealizing ends of a typical neo-impressionist painting, was the result of a scientific reordering, akin of the process of chemical synthesis, of the contemporary world. Likewise, the anarchist faith in the laws of nature as the basis for constructing a social order whose diverse parts were perfectly harmonized correlates with the neo-impressionists' faith in science as the basis for constructing aesthetic harmony, the latter of which, according to Seurat, was the essence of art (Roslak, 2007, pp. 4-5).

L'exemple des réactions et combinaisons des molécules était mobilisé fréquemment pour affirmer qu'entre les éléments moléculaires d'une société (les individus) aussi, l'application de «formules» correctes aurait permis l'établissement de l'harmonie. La technique néo-impressionniste de la division et des touches de couleurs ne serait alors qu'un procédé comparable: l'artiste travaille des "molécules" de couleurs comme le militant et le scientifique social recherchent l'harmonie dans la société (en ce cas métaphore du tableau) en partant des spécificités de l'individu (Roslak, 1991). Cette harmonie chimique et sociale entre particules prend la forme d'une synthèse ; comme l'observe Roslak : "While critics used various terms to describe the expressive quality of a typical neo-impressionist canvas, none occurs more frequently than 'synthesis', a word with both aesthetic and scientific significance in the late nineteenth century " (Roslak, 2007, p. 28).

51 D'après Roslak, ensuite, c'est dans le concept de paysage qu'on trouve des proximités significatives entre la géographie de Reclus et la peinture néo-impressionniste, notamment chez des auteurs comme Signac. « Reclus' geography was more than an effort to understand the earth surface objectively as a disengaged, scientific eye might see. He also understood it subjectively and imaginatively as landscape, often viewed from a distant perspective to conform to an esthetic ideal » (ibid., p. 101). Si d'autres études, comme celles de Denis Cosgrove, ont démontré la puissance du paysage comme dispositif 
à la fois d'imagination et de contrôle sur le monde (ibid., p. 101), pour Roslak l'imagination reclusienne vise à reconstruire à travers le paysage l'idée d'une société harmonieuse, donc libérée de l'oppression et de l'exploitation. Cette idée dialogue sans peine avec le concept de paysage des néo-impressionnistes, visant à reconstruire la même harmonie d'ensemble en partant de parties divisées : de ce point de vue, "neoimpressionist landscapes expressed anarchist ideals » (ibid., p. 8-9).

finalement, souligne plusieurs autres exemples de l'inspiration que les néoimpressionnistes trouvaient dans les ouvrages géographiques de Reclus et Kropotkine, d'un côté par leur intérêt pour une géographie urbaine abordant les problèmes des faubourgs ouvriers (Roslak, 2007, p. 126-132), en particulier chez Signac et Charles Angrand (1854-1926).

De l'autre côté, des peintres comme Henri-Edmond Cross (1856-1910), et Signac lui-même, enferment leurs représentations de l'harmonie dans des paysages méditerranéens (ibid., p. 146-154), en évoquant la représentation anarchiste typique de la Méditerranée en tant que berceau de la civilisation grecque préchrétienne, où les libertaires de l'époque envisageaient l'essor de la pensée libre et laïque, comme le démontrent aussi les écrits de Reclus que nous venons de citer. D'après ce dernier, les îles et presqu'îles de la Méditerranée furent «les replis de cerveau où s'élabore la pensée de l'homme » (Reclus, 1876, p. 47), ce qui explique aussi sa contribution au procès de « l'invention géographique de la Méditerranée » (Deprest, 2002).

Figure 4. Henri-Edmond Cross, Bords Méditerranéens, 1895, Collection particulière.

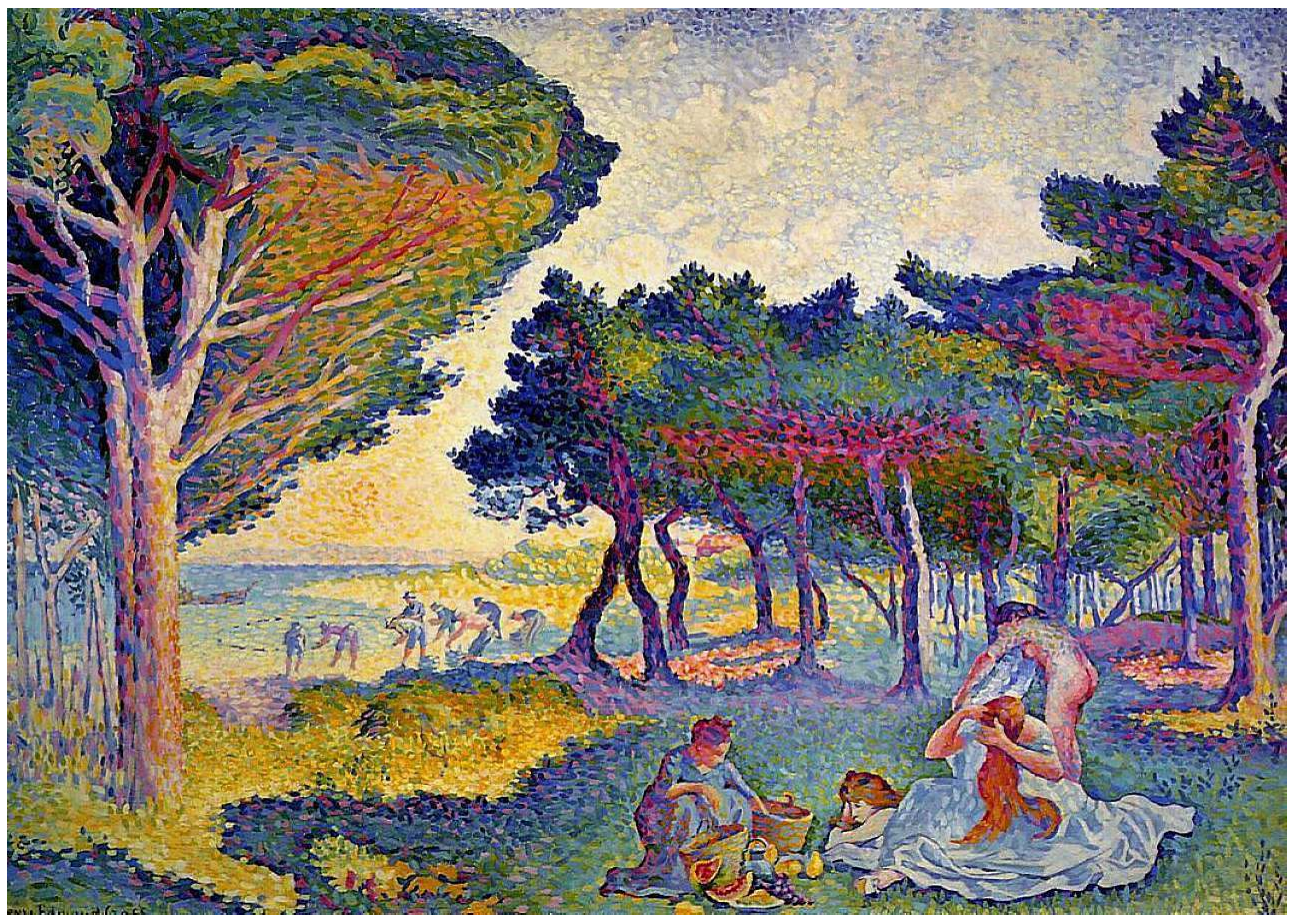

Copyright de l'image : WikiArt.org

Les artistes cités forment des réseaux de sociabilité entre eux et avec le mouvement libertaire de ces années, dans lesquel ils sont presque tous engagés. Marina FerrettiBocquillon a étudié en particulier l'œuvre de Signac et Luce, qui discutaient entre eux de leurs démarches respectives pour la représentation de scènes de vie populaire et de 
symboles de l'action des travailleurs pour détruire la société présente, comme dans le cas du Démolisseur de Signac (Ferretti-Bocquillon, 2010, pp. 20-21).

Ces deux artistes étaient aussi liés aux circuits des artistes et intellectuels libertaires de Bruxelles, en contact avec Reclus et l'Université Nouvelle, comme Émile Verhaeren (1855-1916) et Théo van Rysselberghe (1862-1926) : Luce, en particulier, travailla quelque temps en Belgique (ibid., p. 21). Pour donner un exemple de la circulation internationale de ces artistes et militants, nous pouvons citer la figure de Camille Platteel (1854-1943). Cette dernière, amie de famille des Rysselberghe et enseignante aux écoles communales de Bruxelles, est l'auteure des seules notes survivantes du cours de Géographie comparée que Reclus donnait à l'Université Nouvelle. ${ }^{7}$ En même temps, elle est très liée à Félix Fénéon (1861-1944), figure centrale des échanges entre anarchistes et néoimpressionnistes, qu'elle suit à Paris où elle sert de relais pour ces réseaux, en étant présente «à toutes les manifestations d'avant-garde, au courant de tout » (Halperin, 1991, p. 362).

Camille Pissarro (1830-1905), a été le protagoniste d'une correspondance avec Jean Grave (1854-1939) portant en particulier sur la réalisation de dessins pour financer la presse anarchiste. ${ }^{8}$ Grave, l'un des militants les plus proches de Reclus et Kropotkine, éditeur du journal Le Révolté fondé par eux en 1879 en Suisse et ensuite publié à Paris sous le nom de La Révolte et puis de Temps Nouveaux, témoigne dans ses mémoires du soutien économique que ses initiatives éditoriales recevaient de la part des néo-impressionnistes. Une partie d'entre eux collaborent directement au Temps Nouveaux par la réalisation de vignettes de caractère social, antimilitariste, anticolonialiste. "On pouvait compter sur la bonne volonté de certains dont la réputation n'était pas à faire: Steinlen, Willette, Roubille, Iribe, Grandjouan, Luce, Signac, Agar, Couturier, Angrand, Delaw, Delannoy, Van Dongen, Lebasque, Jossot, Kupka » (Grave, 2009, p. 409).

Selon Grave dans les années 1890, alors que la publication de la Nouvelle Géographie universelle était terminée et que Reclus n'avait plus les moyens financiers de soutenir la revue en payant de sa poche (voir sur cela : Ferretti, 2010), « ce fut Pissarro qui, deux fois, paya nos dettes chez l'imprimeur, déposant mille francs à chaque fois » (Grave, 2009, p. 517). Parmi les moyens de financement de la presse anarchiste il y avait les tombolas, où parmi les primes on pouvait choisir parmi un véritable catalogue d'artistes d'avant-garde, qui se mettaient à disposition pour la cause. "Pour vingt sous on pouvait gagner des tableaux de Angrand, Agar, Bonnard [...] Cross, Mme Couturier, Van Dongen, Delannoy, d'Espagnat, Grandjouan, Hermann-Paul, F. Jourdain, Lebasque, Lefèvre, Manzana, Paviot, Pissarro père, L. Pissarro, Luce, Petitjean, Roubille, Van Rysselbeghe, Raieter, Steinlen, Valloton et Willette. Et je ne cite que les plus connus » (ibid., p. 520).

Comme en témoignent ses archives, Grave était en correspondance suivie avec tous les artistes listés. Signac est l'un des plus sensibles à ses appels pour financer le mouvement anarchiste et illustrer ses journaux (figure 5); le peintre restera pendant plus de trente ans en contact avec Grave, dont il admire «le langage politique net et précis et définitif (...) ; pas des phrases : des idées, des faits $»{ }^{9}$

Figure 5. Pierrefitte-sur-Seine, Centre d'Accueil et de Recherche des Archives Nationales, Fonds IFHS, Dossier 14 AS 184, lettre de Paul Signac à Jean Grave, 10 mars 1902. 


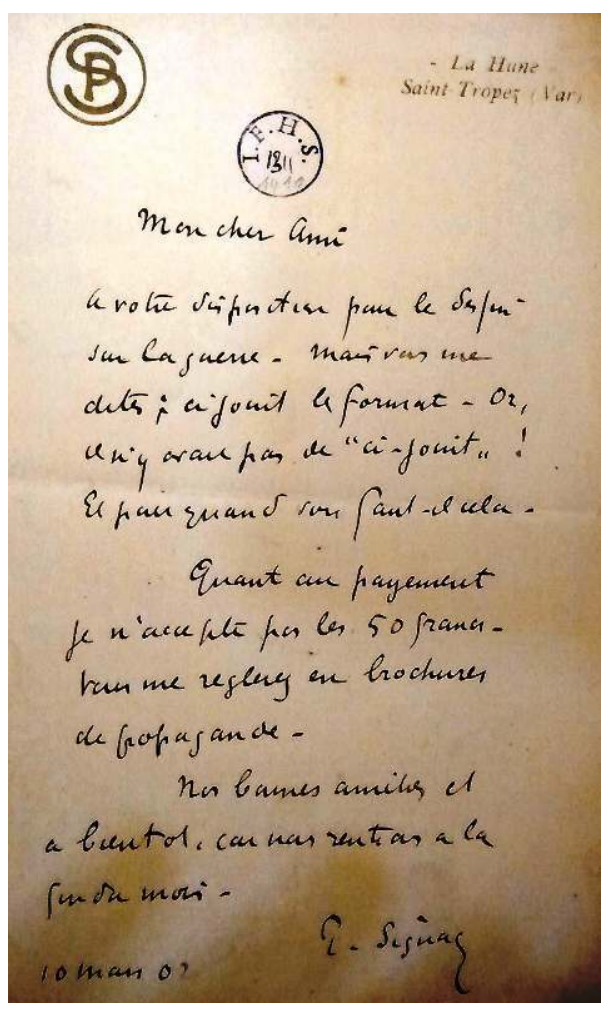

Pissarro, dans ses lettres au fils Lucien, fait état de ses relations d'amitié avec Reclus et Van Rysselberghe. Les trois hommes voyagent quelques jours ensemble dans la Flandre en juillet 1894 (Pissarro, 1988, p. 469) ; d'après Joël Cornuault, ce voyage symbolise une sorte d'unité de la peinture et de la géographie dans le cadre de la même excursion de terrain (Cornuault, 1999).

61 Signalons encore que l'un des gendres de Reclus, William Barbotin (1861-1931), était peintre, bien que de style plutôt conventionnel (il faisait surtout gravures et copies d'œuvres célèbres pour des finalités commerciales). Néanmoins, il collabore à l'illustration des œuvres géographiques de son beau-père et milite quelque temps dans le mouvement anarchiste (Jung, 2013), en réalisant aussi une série de gravures destinées à la vente pour financer les Temps Nouveaux, notamment des portraits d'anarchistes célèbres parmi lesquels Bakounine, Proudhon et Cafiero (Grave, 2009, p. 529).

\section{Conclusion}

Nous pouvons donc conclure que Reclus et les géographes anarchistes ont été fortement concernés par les arts visuels de leur époque, dans le cadre d'une relation constante entre art, géographie et anarchisme, termes qui se sont conditionnés mutuellement, comme on l'a vu dans cet article et dans d'autres travaux ici cités.

Cependant, on peut dire que Reclus, s'il côtoie d'un côté des avant-gardes telles que les néo-impressionnistes et les précurseurs de l'abstraction comme Kupka, conserve de l'autre une conception de l'art qui reste plutôt proche de la démarche de Courbet et Proudhon. Tout en s'occupant personnellement de la construction des systèmes iconographiques de ses ouvrages, le géographe anarchiste se focalise sur la valeur sociale et pédagogique de l'art sans préférer un langage visuel à un autre. 
64 philosophie de la nature telle qu'il la réinterprète dans sa géographie sociale: le monde est l'œuvre d'art, et la métaphore de l'artiste qui travaille ses matériaux sert à définir l'humanité qui embellit le monde, dans la même manière où le réformateur social embellit (c'est-à-dire améliore) la société. L'engagement de Reclus, Perron et d'autres pour la construction d'objets tridimensionnels peut se lire aussi, de ce point de vue, comme la tentative de raccourcir le plus possible la distance entre le monde et sa représentation, le naturel et l'artificiel, l'auteur et l'œuvre.

La contribution de ce travail à la littérature reclusienne est de confirmer l'importance des réseaux dans la production du savoir à la fois scientifique et engagé qui a caractérisé les géographes anarchistes. A travers les exemples présentés, on perçoit l'effet de ces réseaux en tant que lieux et véhicules de production et de diffusion de la connaissance.

En même temps, cette approche de l'art d'Élisée Reclus et de ses collègues éclaircit les procédés par lesquels ils appréhendent la construction d'une démarche heuristique, où l'aspect esthétique est central en tant qu'instrument d'appréhension et de propagation de la science, chez des savants qui n'admettaient pas une séparation rigide entre auteur et spectateur, ni entre maître et élève.

\section{BIBLIOGRAPHIE}

ADAMS M. (2012), « Art, education and revolution: Herbert Read and the reorientation of British anarchism », History of European ideas, 39, pp. 709-728, DOI: 10.180/13825585.2012.736220.

ALAVOINE-MULLER S. (2003), « Un globe terrestre pour l'Exposition Universelle de 1900. L'utopie géographique d'Élisée Reclus ", L'Espace géographique, 2, pp. 156-170.

ANKER V. (1991), Auguste Baud-Bovy (1848-1899), Berne, Benteli.

BESSE J.M. (2003), Face au monde: atlas, jardins, géoramas, Paris, Desclée De Brouwer.

BRUN C. (2014), « Introduction », in RECLUS E., Les grands textes, Paris, Flammarion, pp. 19-52.

CORNUAULT J. (1999), Élisée Reclus étonnant géographe, Périgueux, Fanlac.

DEPREST F. (2002), «L'invention géographique de la Méditerranée : éléments de réflexion », L'Espace Géographique, 1, pp. 73-92.

DUNBAR, G. (1974), « Élisée Reclus and the Great Globe », Scottish Geographical Magazine, 90, 197, pp. 57-66.

FERRETTI-BOCQUILLON M. (2008) (dir.), Georges Seurat, Paul Signac e i neoimpressionisti, Milano, Skira.

FERRETTI-BOCQUILLON M. (2010), dir., Maximilien Luce, néo-impressionniste: rétrospective, Milan, Silvana Editoriale.

FERRETTI F. (2010), « Les Reclus et la Maison Hachette : la première agence de la géographie française ? ", L'Espace Géographique, 3, pp. 239-252. 
FERRETTI F. (2012a), « Aux origines de l'aménagement régional: le schéma de la Valley Section de Patrick Geddes (1925) ", M@ppemonde, 4 http://mappemonde.mgm.fr/num36/articles/ art12405.html

FERRETTI F. (2012b), Élisée Reclus: lettres de prison et d'exil, Lardy, Éditions A la frontière, 2012.

FERRETTI F. (2013), « Géographie, éducation libertaire et établissement de l'école publique entre le $19^{\mathrm{e}}$ et le $20^{\mathrm{e}}$ siècle: quelques repères pour une recherche ", Cartable de Clio, revue suisse sur les didactiques de l'histoire, 13, pp. 187-199.

FERRETTI F. (2014a), Élisée Reclus, pour une géographie nouvelle, Paris, Éditions du CTHS.

FERRETTI F. (2014b), « Pioneers in the History of Cartography: the Geneva map collection of Élisée Reclus and Charles Perron » Journal of Historical Geography, 43, 1, pp. 85-95.

FERRETTI F., MALBURET PH., PELLETIER PH. (2011), « Élisée Reclus et les Juifs : étude géographique d'un peuple sans État », Cybergeo, http://cybergeo.revues.org/index23467.html HALPERIN J. (1991), Félix Fénéon, art et anarchie dans le Paris fin de siècle, Paris, Gallimard.

HEIMBERG C. (1996), L'œuvre des travailleurs eux-mêmes? Valeurs et espoirs dans le mouvement ouvrier genevois au tournant du siècle (1885-1914), Genève, Slatkine.

JUNG D. (2013). Les anarchistes de l'Ile de Ré, Reclus, Barbotin, Perrier et Cie, Saintes, Les éditions libertaires.

LATOUR B. (1987), Science in action: how to follow scientists and engineers through society, Cambridge, Harvard University Press.

LINDSAY J. (1973), Gustave Courbet, his life and art, Somerset, Adams \& Dart.

MENDIBIL D. (2000) «P. Vidal de la Blache, le « dresseur d'images ». Essai sur l'iconographie de la France : Tableau Géographique (1908) », in ROBIC M.-C. (dir.), Le Tableau de la Géographie de la France de Paul Vidal de la Blache : dans le labyrinthe des formes, Paris, CTHS, pp. 78-106.

PELLETIER P. (2013), Géographie et anarchie : Reclus, Kropotkine, Metchnikoff, Paris, Éditions du Monde libertaire.

PROCHASSON C. (2006), « Ni doctrine, ni école, ni mouvement », Arts et sociétés, éditorial du 21 novembre 2006, http://www.artsetsocietes.org/f/f-prochasson.html

ROSLAK R. (1991), « The politics of Aesthetic Harmony: Neo-Impressionism, science, and Anarchism », The Art Bulletin, 73, 3, pp. 381-390.

ROSLAK R. (2006), « Artisans, consumers and corporeality in Signac's Parisian interiors », Art History, 29, 5, pp. 860-886.

ROSLAK R. (2007), Neo-impressionism and anarchism in Fin-de-siècle France: painting, politics and landscape, Aldershot, Ashgate.

SALÉ M.-P. (2002), « Reclus et Kupka: L'Homme est la nature prenant conscience d'elle-même », in BRULLÉ P., SALÉ M.-P., THEINHARDT M., Vers des temps nouveaux: Kupka, œuvres graphiques, Paris, Réunion des Musées nationaux.

SCHLESSER T. (2005), « Le réalisme de Courbet. De la démocratie dans l'art à l'anarchie ", Images Re-vues, http://imagesrevues.revues.org/322

SPRINGER S. (2013), " Anarchism and Geography: a brief genealogy of Anarchist Geographies », Geography Compass, 7, pp. 46-60. 
VUILLEUMIER M. (1996), « André Léo, Malon et le peintre genevois Auguste Baud », Bulletin de l'Association des amis de Benoît Malon, 4, pp. 13-25.

\section{Archives}

Bruxelles - ULB, Archives de l'Université Nouvelle.

Genève - Bibliothèque de Genève, Département des Manuscrits, Archives Baud-Bovy.

Paris - Bibliothèque Nationale de France, Département des Manuscrits Occidentaux, Nouvelles Acquisitions Françaises, 22914, lettres reçues par Élisée Reclus.

Pierrefitte-sur-Seine, Centre d'Accueil et de Recherche des archives Nationales, Fonds IFHS, Dossier 14 AS 184, Correspondances de Jean Grave.

Londres - British Library, Morris Papers vol. VIII, MS 45345; MS 45346.

\section{Sources imprimées}

COURBET G. (1996), Correspondance de Courbet, Paris, Flammarion.

GEDDES P. (1902), « Nature study and geographical education », Scottish Geographical Magazine, 18, pp. 525-536.

GRAVE J. (2009), Mémoires d'un anarchiste, Paris, éditions du Sextant.

KROPOTKIN P. (1910), Champs, usines et ateliers ou l'industrie combinée avec l'agriculture et le travail cérébral avec le travail manuel, Paris, Stock.

MORRIS W. (1884), Art and socialism, a lecture delivered Jan 231884 before the Secular Society of Leicester, London, W. Reeves.

PISSARRO C. (1950), Correspondance de Camille Pissarro, vol. 3, 1891-1894, Paris, Éd. du Valhermeil.

PROUDHON P.-J. (1875), Du principe de l'art et de sa destination sociale, Paris, Garnier.

READ H. (1943), Education through Art, London, Faber and Faber.

RECLUS E. (1866), « Du sentiment de la nature dans les sociétés modernes », La Revue des Deux Mondes, 63, pp. 352-381.

RECLUS E. (1876), Nouvelle Géographie universelle, vol. I, Paris, Hachette.

RECLUS E. (1895a), Projet de construction d'un globe terrestre à l'échelle du cent-millième, Paris, Edition de la «Société Nouvelle».

RECLUS E. (1895b), Université nouvelle de Bruxelles. Séance solennelle de rentrée du 22 octobre 1895. Discours de M. Élisée Reclus, Bruxelles, Imprimerie veuve Ferdinand Larcier.

RECLUS E. (1898), L'évolution, la révolution et l'idéal anarchique, Paris, Stock.

RECLUS E. et al. (1903), « On spherical maps and reliefs » and « Discussion », The Geographical Journal, 3, pp. 290-299.

RECLUS E. (1904). « L’Art et le Peuple », Temps nouveaux, Almanach de la révolution [réimpr. 2012, Écrits sociaux, Genève, Héros-limite, 2012], pp. 140-148.

RECLUS E. (1908), L'Homme et la Terre, vol. VI, Paris, Librairie universelle.

RECLUS E. (1911), Correspondance, vol. I, Paris, Schleicher, 1911.

RECLUS P. (1927), « A Few Recollections on the Brothers Élie and Élisée Reclus », in ISHILL J. (éd.), Élisée and Élie Reclus: in Memoriam, Berkeley Heights, N.J., Oriole Press, art. 25, http:// dwardmac.pitzer.edu/Anarchist_Archives/bright/reclus/ishill/ishill1-25.html 


\section{NOTES}

1. Bibliothèque de Genève (BGE), Département des Manuscrits, Archives Baud-Bovy, Ms., 237, f. 436, lettre d'E. Reclus à A. Baud-Bovy, 30 novembre 1872.

2. Ibid., f.437-438, lettre d'E. Reclus à A. Baud-Bovy, 19 novembre 1874.

3. Bibliothèque Nationale de France Département des Manuscrits Occidentaux, Nouvelles Acquisitions Françaises, f. 16, lettre de D. Baud-Bovy à E. Reclus, 19 septembre 1894.

4. Ibid., f. 467, lettre de F. Kupka à E. Reclus, 4 juin 1905.

5. Bibliothèque Nationale de France, Département des Manuscrits Occidentaux, Nouvelles Acquisitions Françaises (BNF, NAF) 22914, f. 216, lettre de F. Ferrer y Guardia à E. Reclus, 1 juin 1903.

6. Voir les lettres de Kropotkine à Morris et à sa fille, British Library, Morris papers vol VIII, MS 45345, surtout les lettres du 10 janvier 1889 (f. 147), janvier 1889 (f. 148); MS 45346, 25 avril 1889 (f. 54).

7. Bruxelles, Université Libre de Bruxelles, Archives de l'Université nouvelle, I Z 456, Cours de M. Élisée Reclus, Géographie comparée dans le temps et dans l'espace. Notes prises par Mlle Camille Platteel, professeur de géographie aux cours d'éducation B de la Ville de Bruxelles.

8. Pierrefitte-sur-Seine, Centre d'Accueil et de Recherche des archives Nationales, Fonds IFHS, Dossier 14 AS 184, Correspondances de Jean Grave, lettres de C. Pissarro à J. Grave.

9. Ibid., Dossier 14 AS 184, lettre de P. Signac à Jean Grave, 18 novembre 1899.

\section{RÉSUMÉS}

Cet article interroge le rapport à l'art d'Élisée Reclus (1830-1905) et du circuit des géographes anarchistes, affectant à la fois leur approche scientifique et leur engagement politique. Par l'analyse des textes de Reclus sur l'art et des sources primaires permettant de reconstruire ses réseaux, et à l'aide de la littérature internationale portant sur ces thématiques, nous abordons une conception de l'art, de la géographie et de la politique qui envisage le monde comme œuvre d'art, à travers la métaphore apparentant l'artiste qui travaille à ses matériaux pour une finalité esthétique, au scientifique engagé qui travaille à améliorer le monde du point de vue environnemental et social, c'est-à-dire à l'embellir.

This paper deals with the relationship that Élisée Reclus (1830-1905) and the circuit of Anarchist Geographers maintained with Art, which concerned at the same time their approach to Geography and their political engagement. Questioning Reclus' texts on Art and drawing on primary sources on his networks, with the aid of international literature dealing with these topics, I address a relationship among Art, Geography and Politics in which the world was seen as an artwork, by the metaphor comparing the artist working on his materials for an esthetic aim to the engaged scientist working for bettering the world on a social and environmental standpoint, that is to say embellish it. 
INDEX

Mots-clés : Élisée Reclus, Pierre-Joseph Proudhon, néo-impressionnisme, art social, géographie et anarchisme, éducation géographique, naturphilosophie

Keywords : neoimpressionism, social art, geography and anarchism, geographic education

\section{AUTEUR}

\section{FEDERICO FERRETTI}

Docteur en Géographie, membre associé de l'UMR 8504 Géographie-cités, équipe EHGO Épistémologie et Histoire de la Géographie, chercheur au Département de Géographie et Environnement de l'Université de Genève, federico.ferretti@unige.ch 